\title{
Una evaluación de la disponibilidad y la calidad de las estadísticas mexicanas sobre la contaminación del ambiente
}

\section{An assessment of the availability and quality of Mexican statistics on environmental pollution}

\author{
Alfonso Mercado García* \\ Carlos Roberto López Pérez**
}

\section{Resumen}

Es muy poco lo que se sabe sobre la disponibilidad de los datos mexicanos relativos a la contaminación ambiental y menos aún sobre su calidad. El objetivo del presente documento, entonces, es precisamente dar un paso en este conocimiento, mediante una evaluación en temas selectos, siguiendo un método de la escala de Likert. Los temas estudiados son: la contaminación del agua, las emisiones a la atmósfera, los residuos sólidos y la contaminación generada por empresas y hogares. Se detectan mayores limitaciones en la disponibilidad que en la calidad de los datos estudiados. Ello se agrava en tres temas: la relación de la empresa con el ambiente, la de hogar-ambiente y la de residuos sólidos, aunque se observan avances en los dos primeros temas durante el último lustro. Los mayores retos de disponibilidad y calidad se centran en los residuos sólidos. En las causas de estos problemas destaca la falta de buenos registros administrativos y una desvinculación entre la oferta de información estadística y su demanda en los sectores de investigación científica y de toma de decisiones.

Palabras clave: contaminación ambiental; estadísticas del medio ambiente; emisiones a la atmósfera; empresas y medio ambiente; hogares y medio ambiente.

Clasificación JLE: C81, C82, Q56.

* El Colegio de México, A.C. Dirección: Carretera Picacho Ajusco 20, Ampliación Fuentes del Pedregal, 14110, Tlalpan, Ciudad de México. Correo: amercado@colmex.mx ORCID: https://orcid.org/0000-0003-3789-5920

** Consultor. Correo: rlopez386@gmail.com ORCID: https://orcid.org/0000-0001-62560212 


\begin{abstract}
Very little is known about the availability of Mexican data on environmental pollution and even less about their quality. The purpose of this document is therefore to take a step towards increasing this knowledge, through the assessment of selected issues, using a Likert scale method. The topics studied are water pollution, air emissions, solid waste and pollution generated by businesses and households. Greater limitations are detected in the availability than the quality of the data studied. This is particularly noticeable in three issues: the relationship between firms, households and solid waste and the environment, although progress has been observed in the first two in the past five years. The biggest challenges of availability and quality focus on solid waste. As for the causes of these problems, there is a lack of good administrative records and a mismatch between the supply of statistical information and demand for the latter in scientific research and decision-making sectors.
\end{abstract}

Keywords: environmental pollution; environmental statistics; air emissions; business and environment; households and environment.

JEL classification: C81, C82, Q56.

\title{
1. Introducción
}

En una situación de insuficiente disponibilidad de estadísticas, hay costos sociales por las ineficiencias inherentes a esta falta de cifras y por la autoproducción de los usuarios para cubrir los vacíos de datos. Si la calidad de la información estadística no fuese bien atendida, habría costos sociales de desperdicio y de oportunidad. Los dos escenarios juntos plantean un riesgo de doble pérdida en la generación de estadísticas. Por otro lado, la disponibilidad de datos de buena calidad es una condición necesaria, tanto para un diagnóstico realista de problemas sociales o individuales, como para una política eficaz; de manera que la falta de dicha información constituye un vacío que puede frustrar toda aspiración de progreso. Es claro que la oferta de datos y su calidad constituyen un elemento básico y relevante en todo campo de investigación y de toma de decisiones, incluido el tema del medio ambiente y los recursos naturales.

Los antecedentes del desarrollo de esta información estadística son relativamente recientes -apenas tres décadas, en términos generales-, a diferencia del largo recorrido que llevan los datos económicos, demográficos y sociales. En el caso de México, hay pocos temas con una oferta de cifras de larga trayectoria, como las antiguas estadísticas sobre el clima y la atmósfera, algunas de las cuales se remontan a fines del siglo XIX (por ejemplo, el Servicio Meteorológico Nacional se fundó en 1877). También la información sobre el agua tiene largo tiempo, considerando que la Dirección del 
Agua, Tierra y Colonización se creó a principios del siglo XX. En general, la estadística generada en México sobre el ambiente y los recursos naturales es de reciente producción. Es muy poco lo que se sabe sobre la disponibilidad de esta información y, como se verá más adelante, se conoce menos acerca de su calidad. El objetivo del presente documento, entonces, es precisamente dar un paso en este conocimiento.

Aquí no se abarca el amplio conjunto de datos ambientales, sino que se comprende un subconjunto de temas selectos de las estadísticas sobre la contaminación ambiental, con el objetivo de avanzar en el conocimiento de la disponibilidad y la calidad en esos temas. En especial, se examinan las estadísticas sobre la contaminación del agua, las emisiones a la atmósfera, los residuos sólidos y la contaminación generada por empresas y hogares. También se busca dar un paso en la revisión de dichas estadísticas, proporcionando elementos para la elaboración de protocolos para la mejora de las mismas.

Los temas estudiados abarcan gran parte del Marco para el Desarrollo de las Estadísticas Ambientales (MDEA) 2013 de Naciones Unidas, el cual define el contenido de dichas estadísticas mediante seis componentes, entre los cuales nos referimos principalmente a los tres siguientes: a) condiciones y calidad ambiental (datos geoespaciales, físicos y cualitativos, entre los que se encuentran las cifras de características hidrográficas, calidad del agua, condiciones atmosféricas y calidad del aire); b) residuos (datos físicos que incluyen cifras sobre residuos sólidos, descargas contaminantes al agua $\mathrm{y}$ al suelo, y emisiones al aire); c) asentamientos humanos y salud ambiental (cifras geoespaciales y físicas, entre las que se encuentran datos sobre cuestiones ambientales de hogares y empresas, la exposición de la población a contaminantes y riesgos de salud) (Naciones Unidas, 2013).

En la sección 2 se explica el marco conceptual y metodológico del presente estudio. Posteriormente, en la sección 3 se revisan los antecedentes principales sobre el tema en México. Luego, en las secciones 4 a 8 se muestran las principales fuentes estadísticas y se evalúa la disponibilidad y la calidad en cada uno de los cinco temas seleccionados. Finalmente, en la sección 9 se ofrece una breve recapitulación y se presentan las principales conclusiones, con atención especial en los desafíos centrales de mejora.

\section{Conceptos y metodología}

La disponibilidad de estadísticas sobre la contaminación ambiental se conceptualiza aquí como una oferta plena de las mismas, de ágil acceso y con 
facilidades para su uso oportuno y periódico. ${ }^{1}$ Es decir, las estadísticas referidas deben ser generadas y sistematizadas con cierta frecuencia y oportunidad, y estar al alcance para su uso por los demandantes, sobre todo los usuarios que hacen investigación y los que diseñan y evalúan la política pública. Los datos existentes en las dependencias gubernamentales deben quedar fácilmente a la mano del público, con diseños de páginas web amigables para su localización, y sin necesidades burocráticas como las de permisos especiales. Todo ello implica una capacidad de divulgación para hacer "transparente" la información estadística. ${ }^{2}$ Así, dos criterios centrales de la disponibilidad son: $a$ ) una oferta accesible, y b) la oportunidad y periodicidad de su difusión.

En cuanto a la calidad de las estadísticas estudiadas, se concibe aquí como el conjunto de las propiedades inherentes a las mismas que les confieren una capacidad para satisfacer las necesidades de sus usuarios. Dichas propiedades son: el marco conceptual, el diseño muestral, los procedimientos de recolección, procesamiento y tratamiento de la información, el uso de normas, conceptos, marcos ordenadores, clasificadores y catálogos, el propio control de calidad realizado a lo largo del proceso de la producción de estadísticas, la generación de una sola versión del dato (cuidando la diversidad territorial y temporal), así como la credibilidad de las cifras. ${ }^{3}$ En especial, aquí se evalúa la calidad en función de los siguientes cuatro criterios: $a$ ) una sola versión del dato; $b$ ) estadística creíble (clara y fidedigna); c) claridad y fortaleza del marco conceptual y ordenador en el que se basa el dato; ${ }^{4} \mathrm{y} d$ ) rigor de los procedimientos de recolección y tratamiento de las estadísticas. ${ }^{5}$

${ }^{1}$ López Pérez (2014) hace referencia a la suficiencia y la oportunidad del dato, entendiendo por suficiencia la "existencia, relevancia y nivel de cobertura geográfica y temporal", y por oportunidad: la dinámica de la "actualización y difusión."

${ }^{2}$ Hay varios estudios sobre el tema en América Latina y El Caribe, entre los cuales destacan los cuatro siguientes, en orden cronológico: Quiroga, 2005; Schuschny y Soto, 2009; Taboulchanas y Fernández, 2009; Guevara Sanginés y Lara Pulido, 2014.

${ }^{3}$ La calidad de la información básica e indirecta también se ha definido en función de la precisión, la consistencia y el nivel de aplicación del método (véase, por ejemplo, López Pérez, 2014).

${ }^{4}$ Se entiende como marco conceptual la infraestructura de conceptos, metodologías, clasificaciones y estándares aplicados en la generación y sistematización de la información. Todo marco ordenador debe contar con una base conceptual que incorpore las necesidades particulares de análisis y que considere la disponibilidad de información. Han surgido varios marcos ordenadores de la estadística ambiental para estructurar la información (véase Schuschny y Soto, 2009), siendo el PER (explicado en la nota 1) el de mayor difusión mundial, por lo cual nos apoyamos en el mismo.

${ }^{5}$ Una condición fundamental para la alta calidad es tener capacidad de recolección y tratamiento. Hay dos formas de recolección: a) directa, mediante censos y encuestas; y $b$ ) indirec- 
En la evaluación de las estadísticas sobre la contaminación ambiental por cada uno de los temas seleccionados, se consideran observaciones documentadas de los criterios ya explicados, siguiendo una escala de Likert (1932). ${ }^{6}$ Así, se juzga la forma en que se procede para aplicar cada uno de estos criterios, considerando las siguientes cuatro opiniones: $a$ ) pésimo, $b$ ) mal, c) bien, $\mathrm{y} d$ ) excelente. En esta escala, se otorga una calificación de 1 a la primera opinión, 2 a la segunda, 3 a la tercera y 4 a la última. ${ }^{7}$ El método tiene cierto grado de subjetividad, lo cual se tratará de minimizar. ${ }^{8}$ Siguiendo los lineamientos descritos, se llena un formato como el que se presenta en el Cuadro 1, por tema e indicando la opinión sobre cada criterio. Como ejemplo, se presenta en dicho cuadro la evaluación de dos temas. En el tema A se juzga que en la accesibilidad se ha procedido "bien", en tanto que la periodicidad con oportunidad se opina que se hace "mal", y se asignan sus respectivos puntos de calificación.

Con base en estos puntos, se elabora un resumen de evaluación, como se muestra en el Cuadro 2. En el mismo se aprecia que el tema B es mejor evaluado que el A en cuanto a la disponibilidad (un promedio de 3.5 puntos, en comparación con el de calidad, 2.5), pero es peor evaluado que el tema A en lo referente a la calidad ( 2 contra 3 ). Un cuadro de resumen como éste es el que se construirá aquí para cada uno de los temas seleccionados.

\section{Antecedentes}

Los antecedentes sobre el estado que han guardado la disponibilidad y la calidad de las estadísticas en México son de hace una década. El Instituto Nacional de Estadística y Geografía (INEGI) ha llevado a cabo varias re-

ta, a través de registros administrativos. Adicionalmente a la capacidad de solicitar y recolectar la información, la autoridad estadística requiere de una metodología para el tratamiento de las estadísticas y su adecuada integración.

${ }^{6}$ Ésta es una técnica comúnmente utilizada en cuestionarios y encuestas de investigación en varias disciplinas, sobre todo en evaluaciones educativas (especialmente la educación médica y de la salud), estudios de mercadotecnia y en análisis de diversas áreas para la medición de preferencias, actitudes y opiniones. Véase, por ejemplo, Carifio y Perla, 2007; Clason y Dormody, 1994; Sullivan y Artino Jr., 2013; Summers, 1970.

${ }^{7}$ Se optó por una escala Likert con un total de opciones de respuesta en número par (4), en vez de non (5), para evitar un punto medio ("ni bien ni mal", "regular") que podría generar sesgos de tendencia central y resultados ambiguos (Adelson y McCoach, 2010; Carifio y Perla, 2007.

${ }^{8}$ La evaluación de los datos ambientales se ha realizado considerando algunos criterios fundamentales: la competencia institucional (atribuciones y obligaciones de las áreas involucradas), la vigencia o actualidad de la información, la desagregación territorial, temática y temporal, la accesibilidad y la sistematización (periódica y actualizada). 
visiones y evaluaciones de sus estadísticas: en los años 2006, 2008 y 2012. Las dos primeras evaluaciones se llevaron a cabo siguiendo las directrices de los marcos conceptuales y categorías de información de las Naciones Unidas y la OCDE. Se detectaron rezagos en la disponibilidad, al calcularse que la generación de datos ambientales equivalía a $61 \%$ del total ofrecido por la matriz de estadísticas del tema en dichos organismos internacionales. De las categorías del marco ordenador presión-estado-respuesta (PER), ${ }^{9}$ los mayores rezagos se encontraban en las categorías de estado y respuesta; no tanto en la de presión (véase el Cuadro 3).

\section{Cuadro 1}

Formato para evaluar la disponibilidad y la calidad de las estadísticas sobre los temas A y B

\begin{tabular}{|c|c|c|c|c|c|}
\hline Concepto & $\begin{array}{c}1 \\
\text { Pésimo }\end{array}$ & $\begin{array}{c}2 \\
M a l\end{array}$ & $\begin{array}{c}3 \\
\text { Bien }\end{array}$ & $\begin{array}{c}4 \\
\text { Excelente }\end{array}$ & Total \\
\hline \multicolumn{6}{|l|}{$\operatorname{Tema} A$} \\
\hline \multicolumn{6}{|l|}{ Disponibilidad } \\
\hline a) Accesibilidad de los datos & & & $\mathrm{X}$ & & 3 \\
\hline $\begin{array}{l}\text { b) Difusión periódica } \\
\text { y oportuna }\end{array}$ & & $\mathrm{X}$ & & & 2 \\
\hline Suma de los puntos & 0 & 2 & 3 & 0 & 5 \\
\hline \multicolumn{6}{|l|}{ Calidad } \\
\hline a) Un solo dato & & $\mathrm{X}$ & & & 2 \\
\hline b) Cifras creíbles & & & $\mathrm{X}$ & & 3 \\
\hline $\begin{array}{l}\text { c) Fortaleza del marco } \\
\text { conceptual y ordenador }\end{array}$ & & & & $\mathrm{X}$ & 4 \\
\hline $\begin{array}{l}\text { d) Procedimientos } \\
\text { de recolección y tratamiento }\end{array}$ & & & $\mathrm{X}$ & & 3 \\
\hline Suma de los puntos & 0 & 2 & 6 & 4 & 12 \\
\hline
\end{tabular}

${ }^{9}$ El marco PER, basado en Rapport y Friend (1979), fue adoptado por la OCDE a principios de los años 1990 con el objetivo de uniformizar entre sus países miembros el orden de las estadísticas ambientales. Los indicadores de presión se refieren a las fuentes de contaminación y de agotamiento de los recursos (por ejemplo, en el caso del agua, la descarga de residuos peligrosos a cuerpos de agua). Los indicadores de estado incluyen información de la situación y evolución del ambiente (demanda bioquímica de oxígeno en aguas superficiales). Finalmente, los indicadores de respuesta se refieren a estadísticas sobre las medidas que adopta la sociedad (agua residual que recibe tratamiento). Hay derivaciones de este marco, como el de FPEIR (fuerzas motrices- presión- estado- impacto- respuesta) adoptado por la Agencia Europea de Medio Ambiente, Unión Europea, a finales de la década de los 1990. 


\section{Cuadro 1}

(concluye)

\begin{tabular}{|c|c|c|c|c|c|}
\hline Concepto & $\begin{array}{c}1 \\
\text { Pésimo }\end{array}$ & $\begin{array}{c}2 \\
M a l\end{array}$ & $\begin{array}{c}3 \\
\text { Bien }\end{array}$ & $\begin{array}{c}4 \\
\text { Excelente }\end{array}$ & Total \\
\hline \multicolumn{6}{|l|}{ Tema B } \\
\hline \multicolumn{6}{|l|}{ Disponibilidad } \\
\hline a) Accesibilidad de los datos & & & & $\mathrm{X}$ & 4 \\
\hline $\begin{array}{l}\text { b) Difusión periódica } \\
\text { y oportuna }\end{array}$ & & & $\mathrm{X}$ & & 3 \\
\hline Suma de los puntos & 0 & 0 & 3 & 4 & 7 \\
\hline \multicolumn{6}{|l|}{ Calidad } \\
\hline a) Un solo dato & & $\mathrm{X}$ & & & 2 \\
\hline b) Cifras creíbles & & $\mathrm{X}$ & & & 2 \\
\hline $\begin{array}{l}\text { c) Fortaleza del marco } \\
\text { conceptual y ordenador }\end{array}$ & & & $\mathrm{X}$ & & 3 \\
\hline $\begin{array}{l}\text { d) Procedimientos } \\
\text { de recolección y tratamiento }\end{array}$ & $\mathrm{X}$ & & & & 1 \\
\hline Suma de los puntos & 1 & 4 & 3 & 0 & 8 \\
\hline
\end{tabular}

Fuente: Elaboración propia.

\section{Cuadro 2}

Evaluación de la disponibilidad y la calidad de las estadísticas sobre los temas A y B (puntos de la evaluación)

\begin{tabular}{lll}
\hline & \multicolumn{2}{c}{ Tema } \\
\cline { 2 - 3 } Concepto & $\boldsymbol{A}$ & $\boldsymbol{B}$ \\
\hline Disponibilidad & 3 & \\
a) Accesibilidad de los datos & 2 & 4 \\
b) Difusión periódica y oportuna & 5 & 3 \\
Suma de los puntos & 2.5 & 7 \\
Promedio & 2 & 3.5 \\
\hline Calidad & 3 & 2 \\
a) Un solo dato & 4 & 2 \\
b) Cifras crébles & 3 & 3 \\
c) Fortaleza del marco conceptual y ordenador & 12 & 8 \\
d) Procedimientos de recolección y tratamiento & 3.0 & 2.0 \\
Suma de los puntos & & \\
Promedio & 3.0
\end{tabular}

Nota: 1 punto $=$ pésimo; $2=$ mal; $3=$ bien; $4=$ excelente.

Fuente: Elaboración propia. 


\section{Cuadro 3}

Diagnósticos sobre estadísticas e indicadores ambientales de México

\begin{tabular}{|c|c|c|c|c|c|c|}
\hline \multirow[b]{3}{*}{ Categoría } & \multicolumn{3}{|c|}{ Evaluación 2006} & \multicolumn{3}{|c|}{ Evaluación 2008} \\
\hline & \multirow{2}{*}{$\begin{array}{c}\begin{array}{c}\text { Estadísticas } \\
\text { matriz } \\
\text { ONU }\end{array} \\
\text { Cantidad } \\
\text { variables }\end{array}$} & \multicolumn{2}{|c|}{$\begin{array}{l}\text { Disponibilidad } \\
\text { en México }\end{array}$} & \multirow{2}{*}{ 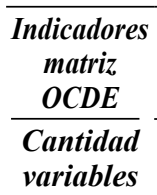 } & \multicolumn{2}{|c|}{$\begin{array}{l}\text { Disponibilidad } \\
\text { en México }\end{array}$} \\
\hline & & $\begin{array}{l}\text { Cantidad } \\
\text { variables }\end{array}$ & $\begin{array}{c}\% \\
\text { México }\end{array}$ & & $\begin{array}{l}\text { Cantidad } \\
\text { variables }\end{array}$ & $\begin{array}{c}\% \\
\text { México }\end{array}$ \\
\hline Presión & 123 & 82 & 66.7 & 38 & 32 & 84.2 \\
\hline Estado & 55 & 32 & 58.2 & 27 & 12 & 44.4 \\
\hline Respuesta & 93 & 52 & 55.9 & 26 & 12 & 46.2 \\
\hline Total & 271 & 166 & 61.3 & 91 & 56 & 61.5 \\
\hline
\end{tabular}

Fuente: López Pérez (2014, cuadro 1, p. 559), basado en confrontaciones hechas por la Dirección de Medio Ambiente del INEGI en los dos años referidos, entre la información disponible y los requerimientos estadísticos de Naciones Unidas (1985) y la OCDE (2005).

La tercera de las evaluaciones referidas (2012) se hizo con motivo de una evaluación a la propuesta de un nuevo marco para la generación de estadísticas ambientales: el Marco para el Desarrollo de las Estadísticas Ambientales (MDEA, o FDES, por sus iniciales en inglés) (véase Naciones Unidas, 2012). Este marco define el alcance y los límites de las estadísticas del medio ambiente en una estructura de seis componentes, desagregados en 61 tópicos y 210 subtópicos, distribuidos en un total de 498 estadísticas ambientales. En la evaluación se encontró que, de estas 498 estadísticas, solamente 195 (equivalente a 39.1\% del total) eran "idénticas" a las definidas por el MDEA; 85 (17.1\%) eran "similares" y 218 (43.8\%) no eran producidas en el país. Es decir, se detectó que aproximadamente dos quintas partes de la gama de estadísticas del MDEA no se ofrecían en México y que un tercio de las disponibles eran solamente "similares" a las del dicho marco, revelando faltas de rigor en la calidad.

Otros elementos de evaluación corroboran una falta de disponibilidad. Un ejemplo es el de la cuenta satélite del medio ambiente, la cual ha logrado progresos en el cálculo de los costos del agotamiento de los recursos naturales a nivel macro, pero todavía falta avanzar en la medición de la interacción de los sistemas económicos y los ecosistemas naturales. Otro ejemplo es el de las estadísticas ambientales del sector agropecuario, uno de los más contaminadores de México (por su emisión de gases de efecto invernadero y sus daños a los cuerpos de agua). Estos datos son insuficientes para la valoración y análisis ambiental del sector, así como para el 
diseño y evaluación de políticas ambientales públicas. Más en específico, también se necesitan mejoras en el inventario de la biodiversidad mediante el monitoreo y la generación de indicadores para una mejor evaluación de los ecosistemas y sus servicios ambientales. Respecto de la deforestación, no hay datos comparables, producidos con un mismo objetivo y que tengan cobertura nacional. Las cifras disponibles son dispares (para un mayor detalle de estas y otras limitaciones, consúltese, por ejemplo, INEGI, 2008, y Mercado García y López Pérez, 2014). Sí ha habido un crecimiento en la disponibilidad, aunque necesita avances adicionales, tanto en lo específico, por tema ambiental, como en lo agregado. ${ }^{10}$ En síntesis, muy poco es lo que se sabe sobre la disponibilidad de esta información estadística y menos aún sobre su calidad.

\section{Contaminación del agua}

Dos importantes fuentes de información estadística en México sobre la contaminación del agua, además de la dotación y distribución del recurso, son: a) la publicación Estadísticas del agua en México, a cargo de la Comisión Nacional del Agua (véase, por ejemplo, Conagua, 2017), que brinda información sobre el estado de las cuencas y los acuíferos del país, la calidad del agua y sus usos; $b$ ) las páginas de internet del INEGI que ofrecen datos del agua (INEGI, 2018a y 2018c). Estas fuentes brindan una amplia cobertura de cifras. En particular, los datos sobre la contaminación o la calidad del agua tratan principalmente sobre los aspectos de monitoreo y evaluación, la calidad del agua subterránea y la del agua en playas, el tratamiento de aguas residuales y riesgos a la salud. Son datos accesibles y su difusión es generalmente periódica y oportuna.

\subsection{La disponibilidad}

a) Oferta accesible. La oferta de los datos sobre la contaminación del agua en México ha mejorado en forma general. Sin embargo, varios de los mismos han sido poco accesibles. Por ejemplo, han faltado datos sobre los costos del servicio y tratamiento de aguas residuales (INEGI, 2008). Todavía es limitada la oferta de datos sobre la calidad del agua, su contaminación,

${ }^{10}$ Por ejemplo, todavía no se conoce bien a bien la tasa del gasto ambiental (público y privado) respecto del producto interno bruto; sólo se tienen datos aproximados y varias estimaciones de organismos internacionales. 
las fuentes de descargas, etc. (contrario a la abundante información sobre la abundancia y escasez del recurso, su agotamiento por regiones y otros datos de stock). Un problema central en estos vacíos es la falta de registros administrativos en los organismos operadores del agua y en los principales usuarios y fuentes de contaminación. El Comité Técnico Especializado ${ }^{11}$ del agua no ha podido actuar con eficiencia contra este problema. Así, se evalúa que en la accesibilidad se ha procedido "mal" (2 puntos).

b) Periodicidad con oportunidad. La periodicidad de estas estadísticas ha sido bien cuidada. Sin embargo, falta la actualización del dato y así el mismo no es tan oportuno. En este aspecto no se ha observado una mejora sustancial. Entonces, se juzga aquí que el criterio de periodicidad con oportunidad en este caso no se ha realizado de manera "excelente", sino "bien" (3 puntos).

\subsection{La calidad}

En la experiencia mexicana sobresale el desarrollo de la calidad estadística sobre la contaminación (y el stock) del agua en los últimos tres lustros. Pero todavía hay varios problemas puntuales por resolver al respecto. Se examinará esta situación enseguida, considerando los cuatro criterios de evaluación que se explicaron en la sección de metodología.

a) Un solo dato. Un desafío importante de la calidad es que no hay uniformidad en cobertura en lo espacial ni en lo temporal, por fuente estadística. ${ }^{12}$ Las fuentes brindan datos con diferentes coberturas. Por ejemplo: varios datos del agua difieren entre la Conagua y el INEGI por diferencias en cobertura temporal y de escala territorial. Debe haber un solo dato en todo el conjunto de la información sobre el tema. Con esta falta de uniformidad, varias estadísticas al respecto llegan a ser confusas e imprecisas. Aunque se ha evolucionado hacia el sistema de información de un solo dato, habiendo actuado de forma adecuada el Comité Técnico Especializado del Agua, todavía queda camino por avanzar en casos puntuales (como el que se acaba de comentar). Así, respecto del criterio "un solo dato," se evalúa que se ha procedido "mal", con un puntaje de 2.

${ }^{11}$ En México, hay varios de estos comités. Ellos se integran por representantes del INEGI y de las autoridades a cargo de los sectores ambientales, como los del agua y de residuos sólidos. Coordinan la producción de información estadística y geográfica de varios temas ambientales y de recursos naturales (Cámara de Diputados, 2014).

${ }^{12}$ Ellas son principalmente la Comisión Nacional del Agua (Conagua), el Instituto Nacional de Estadística y Geografía (INEGI) y la Asociación Nacional de Empresas de Agua y Saneamiento (ANEAS). 
b) Dato creíble. La estadística existente sobre la calidad y la contaminación del agua es creíble (clara y fidedigna), en general, con la observación de que, como se acaba de comentar, en unos casos hay una duplicación de la información que no coincide entre sí (por ejemplo, los datos de agua potable), lo cual deteriora la credibilidad de la estadística. Así, se considera el criterio de "dato creíble" como "bien" atendido (3 puntos).

c) Marco conceptual y ordenador. El modelo PER, explicado en la introducción, es el marco ordenador adoptado por las autoridades productoras de la estadística del agua, principalmente la Secretaría de Medio Ambiente y Recursos Naturales (Semarnat), la Conagua y el INEGI. Las tres instituciones se han basado en los conceptos y lineamientos metodológicos de las Naciones Unidas y de la Organización para la Cooperación y el Desarrollo Económicos (OCDE) como referentes de las actividades de investigación, compilación, generación, y sistematización de estadísticas sobre el agua y otros temas ambientales en México. El marco conceptual y ordenador es una herramienta que ha impulsado la madurez de esta estadística. Por estas razones, se juzga aquí a este criterio como "excelente", con 4 puntos de la escala adoptada.

d) Recolección y tratamiento. Con respecto a los procedimientos de recolección y tratamiento de la información básica, en general son correctos; sin embargo, en casos puntuales se detectan problemas de recolección de los datos de los organismos operadores del agua, lo cual se ha debido a la falta de registros administrativos de los mismos, como ya se ha comentado. La situación no ha cambiado mucho en el tiempo y se percibe cierta descoordinación entre los organismos y las autoridades estadísticas y sectoriales. En el Sistema Nacional de Información Ambiental y de Recursos Naturales se realizan esfuerzos importantes para lograr la integración de la información. Si bien se ha avanzado en el tratamiento de los datos, desafortunadamente no se ha progresado en la recolección por el obstáculo de la falta de registros. En estas circunstancias, se considera que los procedimientos de recolección y tratamiento no se han aplicado en forma excelente, sino "bien" (3 puntos).

\subsection{Síntesis y recomendaciones}

La evaluación de la disponibilidad y la calidad de los datos sobre la contaminación del agua se muestra en la columna 2 del Cuadro 4. Destacan dos resultados: a) la forma de proceder en materia de la disponibilidad resulta evaluada en promedio cerca de "mal" (no alcanza a ser "bien"), y b) aunque 
la oferta estadística es limitada, la calidad de los datos existentes se evalúa como "bien" atendida, en promedio. Así, la evaluación sugiere mayores problemas de disponibilidad.

Para avanzar en la disponibilidad de esta estadística, se sugiere que las autoridades se acerquen más a los usuarios (sobre todo a los diseñadores y evaluadores de políticas públicas del agua). Faltaría evaluar la factibilidad técnico-financiera, ya que ello significaría una mayor necesidad de recursos humanos y de presupuesto. También se recomienda cubrir vacíos de información específicos, como los ya señalados. Finalmente, se recomienda desarrollar un sistema de registros, en especial en el caso de los organismos operadores del agua.

Respecto de la calidad de las estadísticas, hay dos desafíos centrales que afronta en su mejoramiento: a) generar y difundir una sola versión del dato en los diversos aspectos del tema, y b) mejorar y aplicar con más rigor los procedimientos de recolección y tratamiento de las estadísticas. En atención a estas observaciones, es preciso producir una base de datos común entre Conagua, Semarnat e INEGI para avanzar hacia sistemas de información unificados y más automatizados. En esta línea de acción se debe compatibilizar la información estadística del agua con las cuentas nacionales ecológicas. Es recomendable también mejorar la medición del monitoreo de la calidad del agua.

\section{Emisiones a la atmósfera}

Siendo los datos sobre la atmósfera y el clima los de mayor historia en la estadística ambiental mexicana, se han requerido más cifras al respecto en el último decenio para atender el problema del cambio climático, sobre lo cual México ha hecho un progreso apreciable. Destacan las siguientes dos fuentes de estadística: a) Instituto Nacional de Ecología y Cambio Climático (INECC), Inventario Nacional de Emisiones de Gases de Efecto Invernadero (por ejemplo, INECC, 2018), con registros y cálculos de las emisiones antropogénicas de gases y compuestos de efecto invernadero y de la absorción por los sumideros en México, por principales fuentes de emisión y por ecosistema de captura. b) La página de internet de la Semarnat (2018b), en la parte de "Consulta temática" sobre atmósfera, en la cual se ofrecen datos sobre calidad del aire, gases y compuestos de efecto invernadero, y el ozono estratosférico. ${ }^{13}$

${ }^{13}$ También es preciso citar un trabajo de Galindo (2009) y las siguientes dos publicaciones de la Semarnat: Versión de difusión del Programa Especial de Cambio Climático 2014- 


\subsection{La disponibilidad}

a) Oferta accesible. El acceso a los datos es bueno en general, gracias a un impulso prioritario a esta información y a los trabajos del Comité Técnico Especializado. Adicionalmente, ha ayudado una buena disposición de las fuentes informativas a colaborar con sus estadísticas primarias. Sin embargo, falta todavía una mayor integración entre los aspectos científicos, estadísticos y geográficos. Además, como sostiene Mediavilla Sahagún (2014), hay limitaciones en el inventario de emisiones a la atmósfera, ya que éste no cuenta con suficiente desagregación sectorial ni buena cobertura regional. Con base en lo observado, la oferta estadística de este tema se considera aquí con una accesibilidad que todavía se atiende "mal" (2 puntos).

b) Periodicidad con oportunidad. Los datos de emisiones a la atmósfera tienen buena frecuencia y son distribuidos con oportunidad. Esta situación se ha venido mejorando gracias a un adecuado trabajo interinstitucional para estas estadísticas de alta prioridad y una alta disposición a colaborar con la información. Así, la periodicidad con oportunidad de éstas se evalúa como "excelente" (4 puntos).

\subsection{Calidad}

La estadística ha observado un rápido desarrollo con mayor coordinación entre instituciones, asignándole alta prioridad. En general, los cuatro aspectos considerados se evalúan bien.

a) Un solo dato. La estadística sobre emisiones a la atmósfera presenta una sola versión de los datos, gracias a los trabajos del Comité Técnico Especializado, salvo unos casos en los que hay distintas definiciones del mismo concepto por diversas unidades productoras del dato (véase Mediavilla Sahagún, 2014). Falta que esto se resuelva estrictamente con una sola definición. Por eso, se considera "bien" el criterio de "un solo dato" (3 puntos).

b) Dato creíble. Se ha avanzado para generar estadísticas fidedignas y claras, habiendo tenido un impulso prioritario. Pero todavía hay algunos desafíos por vencer. Uno de éstos consiste en que las cifras de los eventos climáticos extremos proyectados a nivel regional han aparecido con errores y omisiones, generando incertidumbre al tratar de usarlos. Además, hay dudas

2018 (Semarnat, 2014), y Cambio climático. Ciencia, evidencia y acciones (Semarnat, 2009). Estos no son medios de difusión de estadísticas, pero contienen datos y cálculos de interés para el diseño de políticas. 
fundadas sobre el valor específico de varios parámetros económicos. Esto mina la credibilidad del dato. En estas circunstancias, la credibilidad de la información ha resultado afectada adversamente. Es preciso reconocer que, en un panorama amplio, la credibilidad ha crecido gracias a una adecuada relación interinstitucional. Por todas estas razones, la forma de proceder respecto de este criterio se juzga en general "bien" (3 puntos).

c) Marco conceptual y ordenador. El marco ordenador adoptado, PER, es bueno y adecuado; sin embargo, hay algunos problemas conceptuales y de definiciones, los cuales se asocian a la falta de madurez de estas estadísticas y su complejidad. Por ejemplo, como ya se comentó, falta uniformidad en las definiciones de los productores de la estadística, haciendo casi imposible la comparación de cifras entre los mismos, por lo que es preciso mejorar la base conceptual y las categorías del marco ordenador. También se reconoce que se han logrado grandes avances. En tales circunstancias, este marco se considera "bien" atendido (3 puntos).

d) Recolección y tratamiento. Los procedimientos de recolección y tratamiento de esta información son buenos, aunque ya se comentaba que hay dificultades respecto de los eventos climáticos y externos por región, así como de los parámetros económicos. También hay dificultades de recolección por la dinámica dispersión de las fuentes emisoras de gases efecto invernadero. La atención a estos desafíos ha sido positiva y con cierta evolución. Se juzga que estos procedimientos se han hecho "bien" (3 puntos).

\subsection{Síntesis y recomendaciones}

La columna 3 del Cuadro 4 presenta las evaluaciones sobre este tema, entre las que llama la atención la baja puntuación de la accesibilidad a los datos y la alta calificación de la periodicidad y la disponibilidad. Es preciso contar con una oferta más accesible. Similarmente al caso de las cifras del agua, en el caso de las estadísticas de emisiones a la atmósfera también se recomienda acercarse a los usuarios finales; es decir, "subir la información" para activar un círculo virtuoso de datos, conforme a los ejes fundamentales del Programa Especial de Cambio Climático. Estos ejes son: a) riesgos y fenómenos extremos, $b$ ) observación del fenómeno, $c$ ) información sectorial, $d$ ) modelos y escenarios, $e$ ) comunicación y educación, $f$ ) impacto en ecosistemas, $g$ ) impactos socioeconómicos. Además, como propone Mediavilla Sahagún (2014), un inventario de emisiones "paralelo", con base en el ya realizado, que contenga una desagregación por tipo de combustible utilizado y otra por rama industrial, como por ejemplo, la acerera, la cementera, la 
química, la de energía eléctrica y la petrolera, las cuales son las que emiten más gases de efecto invernadero. Para llenar vacíos de información específicos, como el correspondiente a los eventos naturales extremos por regiones, se sugiere que el Comité Técnico Especializado del tema atienda este punto en su agenda de trabajo.

En cuanto a la calidad, es preciso seguir avanzando, para lo cual se sugiere que el INEGI, como autoridad central de las estadísticas, y el Comité Técnico Especializado, con apoyo o asesoría de organismos internacionales, perfeccionen los procedimientos de recolección y tratamiento, el marco conceptual y ordenador, y la claridad de los datos, especialmente los más novedosos y complejos.

\section{Residuos sólidos}

Éste es un tema en el que han participado varias autoridades gubernamentales de distintos sectores, como el Consejo Nacional de Población (dependiente de la Secretaría de Gobernación), la Secretaría de Desarrollo Social, el INECC y el INEGI. Las principales fuentes de datos son: $a$ ) en la página del INEGI (2018a), la sección sobre residuos sólidos, con información sobre la generación, composición y gestión de los residuos sólidos que se originan en las actividades domésticas y comerciales de las localidades; $b$ ) el Sistema de Consulta de Estadísticas Ambientales del INEGI (2018c), en donde se ofrece un banco de datos sobre agua y residuos sólidos; c) el Sistema de Información Ambiental y de Recursos Naturales de la Semarnat (2018b), con datos sobre residuos peligrosos (cifras de su generación, movimientos transfronterizos y manejo) y residuos sólidos urbanos (generación, recolección, disposición y gestión).

\subsection{La disponibilidad}

México todavía no cuenta con suficiente disponibilidad de estadísticas sobre la generación de residuos sólidos, ni sobre su tratamiento ni su disposición final. A falta de registros completos y confiables, gran parte la información estadística del tema se calcula de manera indirecta.

a) Oferta accesible. El acceso a esta información estadística es limitado. No ha habido los suficientes registros administrativos. Además, falta una adecuada coordinación interinstitucional, pues la existente ha dado muestras de no ser efectiva. No ha habido evolución hacia una mejora. En- 
tonces, se juzga que se procede "mal" (2 puntos) en la atención a la accesibilidad de la oferta.

b) Periodicidad con oportunidad. Ni la frecuencia ni la oportunidad de estos datos han sido de calidad. Los datos sobre residuos peligrosos son especialmente muy dispares y no siguen una secuencia año con año. Por ello, la oportunidad y periodicidad se juzga que se realizan "mal" ( 2 puntos).

\subsection{La calidad}

La estadística de residuos sólidos es una de las de mayor madurez en México. Sin embargo, afronta enormes problemas de calidad, principalmente porque se produce indirectamente y es poco confiable. En efecto, es indirecta ya que gran parte es calculada con fuentes externas; es decir, a partir de estimaciones y criterios de autores extranjeros y con referencia a los países del primer mundo. Además, es poco confiable y de alta incertidumbre, pues en su mayoría corresponde a fuentes diversas y descoordinadas, no cumple con requisitos mínimos de verificación y no ofrece una versión única de las estadísticas. Se observan incongruencias en los datos sobre residuos peligrosos, más que en los casos de los residuos sólidos urbanos, los mineros y los de manejo especial.

a) Un solo dato. Esta estadística no ofrece una versión única de los datos. Al respecto, la organización interinstitucional para generar y difundir estas estadísticas no ha funcionado tan bien como en otros temas. Entonces, se opina que este criterio se atiende "mal" (2 puntos).

b) Dato creíble. Como ya se dijo, la captación es pobre y se recurre a cálculos "indirectos", de manera que al dato le falta credibilidad. En tales circunstancias, el criterio de "dato creíble" de esta estadística se juzga que se lleve a cabo "mal" (2 puntos).

c) Marco conceptual y ordenador. El marco ordenador es el PER, y en términos generales es bueno. Es preciso reconocer que en este aspecto ha mejorado la estadística y que la coordinación interinstitucional ha servido para ello. Sólo habría que atender algunos problemas de definición y conceptuales, en especial sobre los residuos peligrosos. Así, se evalúa "bien" este marco (3 puntos).

d) Recolección y tratamiento. Con respecto a los procedimientos de recolección y tratamiento, se detectan problemas, especialmente en lo que se refiere a los residuos industriales peligrosos. A falta de una buena recolección, se elaboran estimaciones indirectas, como ya se comentó. Hay cierta cultura de registros, sobre todo de los residuos industriales peligrosos y los 
de manejo especial, pero falta incrementarla. Los avances en el registro de datos y captación han sido lentos. Por eso, estas cifras se han calculado en formas indirectas. Por estas razones, se evalúa el criterio de recolección y tratamiento de estos datos como "mal" atendido ( 2 puntos).

\subsection{Síntesis y recomendaciones}

El cuidado de la disponibilidad y de la calidad de los datos sobre residuos sólidos es evaluado en promedio como "mal" llevado a cabo (columna 4 del Cuadro 4). Es necesario generar estadísticas de residuos sólidos en forma directa y así mejorar la disponibilidad. Para ello, es preciso aprovechar los datos del sistema de normas sobre residuos, incorporar los datos de residuos al sistema de cuentas nacionales y aplicar una encuesta nacional especializada en el tema.

La evaluación de la calidad de estas cifras es baja. Tan sólo se escapa de tal evaluación el criterio del marco conceptual y ordenador. Como recomendación central, se sugiere que las autoridades emprendan un sistema estadístico de alta calidad que genere datos en forma más directa. Esta estrategia debe partir del fomento del desarrollo de registros administrativos sistemáticos entre quienes administran las actividades de residuos sólidos.

\section{Emisiones contaminantes de las empresas}

La estadística sobre las emisiones contaminantes a la atmósfera, a cuerpos de agua y al suelo, constituye un tema que entró muy recientemente en la agenda de la autoridad estadística mexicana, mucho después que otros temas ambientales. La producción de estos datos ha observado un rápido desarrollo. No hay una fuente exclusiva sobre empresa y ambiente, sino secciones de publicaciones y páginas de internet con estadísticas nuevas al respecto. Destacan las siguientes dos páginas de internet como fuentes estadísticas: la de datos sobre ecología del INEGI (2020) y la del Sistema Nacional de Información Ambiental y de Recursos Naturales, de Semarnat (2018b). En la primera se ofrecen cifras sobre los costos totales por agotamiento y degradación ambiental como proporción del PIB, producto interno neto ajustado ambientalmente, y gasto en protección ambiental como proporción del PIB (datos de 2007 a 2016). En la página de la Semarnat (2018b), en su sección de consulta temática, hay datos sobre riesgo ambien- 
tal, procuración de justicia ambiental, industria y medio ambiente, cuentas ambientales, turismo y transporte. En este sitio de internet se difunden datos sobre fuentes de contaminación ambiental de competencia federal, auditoría ambiental, e impactos ambientales de sectores productivos (manufacturero, petrolero, turismo y transporte).

\subsection{La disponibilidad}

a) Oferta accesible. La oferta de estos datos ha progresado en los últimos lustros. Se han logrado avances a partir del Registro de Emisiones y Transferencia de Contaminantes (Semarnat 2018a) y, sobre todo, más recientemente, en las páginas de internet del INEGI (2020) y de la Semarnat (2018b) descritas antes. Adicionalmente, ha habido intentos de realizar una encuesta especial sobre la relación empresa-ambiente (primero, la Encuesta Nacional de Gasto y Gestión Ambiental, y luego, la Encuesta Nacional de Unidades Económicas y Medio Ambiente). No se ha logrado dicha encuesta, posiblemente por razones de presupuesto. Pese a los avances mencionados, hay todavía vacíos en la oferta de estadísticas, por lo cual, se considera que fue "mal" atendido (2 puntos).

b) Periodicidad con oportunidad. Las estadísticas de la relación empresa-ambiente han mejorado su oportunidad, pero todavía faltan mejoras adicionales. Por ello, se evalúa como "mal" realizadas (2 puntos).

\subsection{La calidad}

Esta estadística es novedosa en México y tiene la complejidad de ser transversal entre el sector económico y el ambiental. Estas características y la falta de registros ambientales -especialmente en el sector de empresas de tamaños micro, pequeño y mediano- han minado la claridad y la credibilidad del dato. Estos son desafíos centrales para el caso.

a) Un solo dato. La estadística se difunde con una sola versión y por ello es evaluada como "excelente" (4 puntos).

b) Dato creíble. Falta credibilidad a los datos, de manera que se evalúa esta área como "mal" atendida ( 2 puntos). Esta debilidad se debe a la poca disposición a colaborar con información de parte del sector empresarial y su falta de un adecuado sistema contable y de registros ambientales. A esto se agrega el hecho de que esta información es compleja y novedosa. Afortunadamente, se observa un progreso en la resolución de estos problemas. 
c) Marco conceptual y ordenador. El marco conceptual y ordenador es adecuado: es el PER. Si bien en general el marco es bueno, hay debilidades y fallas conceptuales puntuales. Este es el caso, por ejemplo, del concepto de gasto ambiental, el cual no se restringe al presupuesto gubernamental que cada país dedica a ese fin, sino también el gasto del sector privado con propósitos ambientales (y aun el gasto de los ciudadanos consumidores -los hogares- para cuidar el ambiente). Así, una pregunta que está pendiente de respuesta por falta de datos es: ¿cuál es el impacto anticontaminante de este gasto? Otro problema conceptual concierne a la inversión anticontaminante de las empresas. Falta precisión en su concepto y, por ende, lo que debe medirse. Estos problemas conceptuales han venido atendiéndose en los últimos años, pero todavía persisten varios problemas puntuales de este tipo. Por todo lo anterior, el marco se considera en general como "bien" realizado (3 puntos).

d) Recolección y tratamiento. Respecto de los procedimientos de regulación y tratamiento, las autoridades estadísticas a cargo del tema han tenido que lidiar con esta información compleja y novedosa, y con una falta de registros empresariales sobre sus emisiones y acciones ambientales, sobre todo en el caso de las empresas de tamaños micro, pequeño y mediano, las cuales cubren gran parte del sector empresarial. Por ello se asigna a los procedimientos de regulación y tratamiento una evaluación de "mal" atendidos (2 puntos).

\subsection{Síntesis y recomendaciones}

La estadística sobre la contaminación de las empresas tiene en promedio una evaluación de haber procedido "mal" en la disponibilidad. En cuanto a la calidad, resulta casi "bien", siendo sus debilidades la credibilidad de las cifras, la periodicidad con oportunidad de su difusión y los procedimientos de recolección y tratamiento (en especial, hay irregularidades de registros) (véase la columna 5 del Cuadro 4).

Se sugiere adoptar una estrategia para ampliar la oferta de datos y elevar la calidad, en especial lo fidedigno y claro del dato. Para mejorar lo fidedigno y lo claro de esta estadística, se propone una estrategia de dos caminos paralelos: a) Acercarse más a la demanda (para satisfacer las necesidades de los principales usuarios) y, al mismo tiempo, guiarse por la oferta (para seguir al exterior, considerando las directrices principalmente de $\mathrm{Na}$ ciones Unidas y la OCDE). Para ello se recomienda reactivar el proyecto de la Encuesta Nacional de Unidades Económicas y Medio Ambiente, y for- 
talecer los Indicadores de Desempeño Ambiental y el RETC. b) Mejorar la coordinación entre las autoridades que cuentan con datos sobre la relación ambiente-empresa. Para este efecto, el INEGI cuenta con la facultad para hacerlo, establecida en la Ley del Sistema Nacional de Información Estadística y Geográfica, como un ente coordinador y normativo, mientras que la Semarnat, actuando como autoridad ambiental, debe acompañarlo y definir las necesidades de información ambiental, a partir de la evaluación existente en el país y la requerida no sólo a nivel nacional, sino internacional, para efecto de cumplir con los compromisos del país en la materia y avanzar en la comparabilidad internacional.

Adicionalmente, se sugiere fortalecer e impulsar mejoras en los instrumentos de dominio propio, concretando un proyecto de encuesta especial en esta temática. También sería valioso fomentar la concientización de muchas empresas en torno al uso de información ambiental como una herramienta esencial para su gestión.

\section{Emisiones contaminantes de los hogares}

Éste es otro tema que se incorporó a la agenda de la autoridad estadística hace relativamente poco tiempo. Se observa un rápido avance en la producción de estadísticas al respecto. Las principales fuentes son: a) La página de estadísticas sobre el medio ambiente de INEGI (2018a), con información sobre las prácticas o acciones que se realizan en las unidades familiares en torno al manejo de la basura o residuos, el transporte y la movilidad, así como en relación con los estilos de vida y pautas de consumo; todo ello en conexión con la degradación de los recursos naturales. b) Dicha página de internet se basa en gran medida en el Módulo de Hogares y Medio Ambiente de INEGI (2018b). c) La página de internet del Sistema Nacional de Información Ambiental y de Recursos Naturales, de la Semarnat (2018b), con información sobre localidades con 20 y más viviendas por destino de descarga y por disponibilidad del servicio público de limpia.

\subsection{La disponibilidad}

a) Oferta accesible. Hay desafíos similares a los de la estadística empresa-ambiente: una falta de registros en gran cantidad de hogares y una compleja transversalidad temática e institucional. El INEGI ha avanzado mediante el lanzamiento de un módulo de hogares y ambiente en el Cen- 
so de Población y Vivienda (véase INEGI, 2018a, 2018b), la aplicación de una encuesta sobre hogar-salud (con cierta información ambiental) y la Encuesta Nacional de Hogares y Medio Ambiente, puesta en marcha en 2019, además de la página de internet de la Semarnat (por ejemplo, véase Semarnat, 2018a, 2018b). Pero hay todavía vacíos estadísticos importantes en el tema, como, por ejemplo, datos relativos a la reducción, los de daños a áreas naturales, descargas contaminantes al suelo y a cuerpos de agua, así como abatimiento de emisiones a la atmósfera. En estas condiciones, al momento de escribir este documento, la accesibilidad de datos se juzga todavía como "mal" llevada a cabo (2 puntos).

b) Periodicidad con oportunidad. Tanto la frecuencia como la oportunidad de estos datos han tenido problemas importantes, aunque se ha avanzado recientemente. Así, el criterio de periodicidad con oportunidad de los datos se evalúa como "mal" atendido ( 2 puntos).

\subsection{La calidad}

a) Un solo dato. Esta estadística se difunde en forma bien coordinada, especialmente entre el INEGI y la Semarnat, con una sola versión; por ello es evaluada al respecto como "excelente" (4 puntos).

b) Dato creíble. Falta credibilidad a los datos por una limitada capacidad de los hogares para colaborar con información y hay falta de registros. A esto se agrega el hecho de que esta información es compleja y novedosa. Afortunadamente, se observa un progreso en resolver estos problemas. Al momento, se considera el criterio "dato creíble" como "mal" atendido (2 puntos).

c) Marco conceptual y ordenador. El marco ordenador es PER y se considera adecuado. Sin embargo, hay debilidades conceptuales, destacando las definiciones de gasto ambiental de los hogares y el "comportamiento" ambiental de éstos. Estas debilidades han venido atendiéndose en los últimos años, pero todavía persisten varios problemas de este tipo. Por eso se evalúa aquí como un marco "bien" realizado (3 puntos).

d) Recolección y tratamiento. Aunque las autoridades estadísticas han tenido que lidiar con una falta generalizada de registros, han venido mejorando los procedimientos de regulación y tratamiento. Se juzga que estos procedimientos, sobre todo los de recolección, son en general "bien" hechos (3 puntos). Es preciso reconocer que se ha estado avanzando en esta área, con el buen papel desempeñado por las instancias de coordinación interinstitucional. 


\subsection{Síntesis y recomendaciones}

La estadística sobre la relación hogar-ambiente resulta con una calificación promedio de haber procedido "mal" en la atención a la disponibilidad y "bien" en materia de la calidad (véase la columna 6 del Cuadro 4).

Para mejorar la disponibilidad, es recomendable trazar una estrategia que fortalezca el desarrollo de las estadísticas sobre hogar-ambiente, de forma que se capten datos sobre reutilización, reciclamiento y reducción del consumo de agua ("las tres $r$ ") y sobre los factores escala, composición y tecnología, que impactan la explotación del recurso. Adicionalmente, se recomienda incorporar información cualitativa con una adecuada coordinación entre los ámbitos ambiental, demográfico y económico. También se sugiere abordar el tema de la migración, el cual es central en el comportamiento de los hogares y es parte de la nueva agenda global.

\section{Conclusiones}

Con base en los resultados de la presente evaluación, siguiendo una escala Likert, se detectan mayores limitaciones en la disponibilidad que en la calidad de los datos estudiados. En la Gráfica 1, la línea de la disponibilidad (con valores de 2.0 a 3.0, promediando 2.8) se encuentra más alejada del límite de excelencia (4.0 puntos) que la línea de la calidad (entre 2.3 y 3.0, con un promedio de 3.1). En la misma gráfica se observa que esta brecha se expande (es decir, se agrava) en tres de los temas estudiados: la relación de la empresa con el ambiente, la de hogar-ambiente y la de residuos sólidos, aunque se observan avances en los dos primeros temas durante el último lustro. Los mayores retos de disponibilidad y calidad se centran en los residuos sólidos.

Además del problema de transversalidad de los temas estudiados y las deficiencias en la coordinación interinstitucional para medir algunos fenómenos ambientales en forma sistemática (por temas, geográficamente y en tiempo), en las causas de estos problemas destacan, por un lado, la carencia de una cultura de registros, no sólo en el hogar, sino también en las empresas, incluso en el gobierno; y, por otro, una desvinculación entre la producción de la información estadística y su demanda en los sectores de investigación científica y de toma de decisiones. Estas dos causas limitan tanto la disponibilidad como la calidad de los datos. 


\section{Cuadro 4}

Evaluación de las estadísticas mexicanas sobre la contaminación ambiental en los temas seleccionados (puntos de la evaluación)

\begin{tabular}{|c|c|c|c|c|c|c|}
\hline $\begin{array}{l}\text { Concepto } \\
\text { (1) }\end{array}$ & $\begin{array}{c}\text { Agua } \\
\text { (2) }\end{array}$ & $\begin{array}{l}\text { Emisiones } \\
\text { a la } \\
\text { atmósfera } \\
\text { (3) }\end{array}$ & $\begin{array}{l}\text { Residuos } \\
\text { sólidos } \\
\text { (4) }\end{array}$ & $\begin{array}{c}\text { Contam. } \\
\text { por la } \\
\text { empresa } \\
\text { (5) }\end{array}$ & $\begin{array}{l}\text { Contam. } \\
\text { por el } \\
\text { hogar } \\
\text { (6) }\end{array}$ & $\begin{array}{c}\text { Promedio } \\
\text { (7) }\end{array}$ \\
\hline \multicolumn{7}{|l|}{ Disponibilidad } \\
\hline $\begin{array}{l}\text { a) Accesibilidad } \\
\text { de los datos }\end{array}$ & 2 & 2 & 2 & 2 & 2 & 2.0 \\
\hline $\begin{array}{l}\text { b) Difusión } \\
\text { periódica } \\
\text { y oportuna }\end{array}$ & 3 & 4 & 2 & 2 & 2 & 2.6 \\
\hline $\begin{array}{l}\text { Suma de los } \\
\text { puntos }\end{array}$ & 5 & 6 & 4 & 4 & 4 & 4.6 \\
\hline Promedio & 2.5 & 3.0 & 2.0 & 2.0 & 2.0 & 2.3 \\
\hline \multicolumn{7}{|l|}{ Calidad } \\
\hline a) Un solo dato & 2 & 3 & 2 & 4 & 4 & 3.0 \\
\hline b) Cifras creíbles & 3 & 3 & 2 & 2 & 2 & 2.4 \\
\hline $\begin{array}{l}\text { c) Fortaleza del } \\
\text { marco concep- } \\
\text { tual y ordenador }\end{array}$ & 4 & 3 & 3 & 3 & 3 & 3.2 \\
\hline $\begin{array}{l}\text { d) Procedi- } \\
\text { mientos de } \\
\text { recolección y } \\
\text { tratamiento }\end{array}$ & 3 & 3 & 2 & 2 & 3 & 2.6 \\
\hline $\begin{array}{l}\text { Suma de los } \\
\text { puntos }\end{array}$ & 12 & 12 & 9 & 11 & 12 & 11.2 \\
\hline Promedio & 3.0 & 3.0 & 2.3 & 2.8 & 3.0 & 2.8 \\
\hline
\end{tabular}

Nota: 1 punto $=$ pésimo; $2=$ mal; $3=$ bien; $4=$ excelente.

Fuente: Elaboración propia.

Es preciso elevar la disponibilidad y seguir con mejoras continuas de calidad en la generación de datos ambientales para abatir el riesgo de doble pérdida (costos sociales), comentado en la introducción de este documento. Ciertamente, la oferta estadística de las instituciones nacionales que son responsables de las mismas (como el INEGI, la Conagua y la Semarnat) se ha acercado a la amplia gama de datos establecida por los organismos internacionales, pero falta ajustarse a: 1) los principales demandantes, y 2) la organización de los datos básicos, primarios, con buenos registros 
sistemáticos. Derivado de esos dos caminos, podría reconsiderarse el desarrollo de nuevas encuestas, módulos de las encuestas o de los censos existentes y otros instrumentos de captación de datos. Dichos elementos se sugieren aquí como centrales para una estrategia de mejora de la información estadística sobre la contaminación ambiental. También se recomienda ensanchar la coordinación interinstitucional más allá de los organismos gubernamentales, incorporando a los principales usuarios de esta información que se encuentran en la investigación científica y en la toma de decisiones públicas y privadas.

\section{Gráfica 1}

Evaluación de la disponibilidad y la calidad de las estadísticas sobre contaminación ambiental

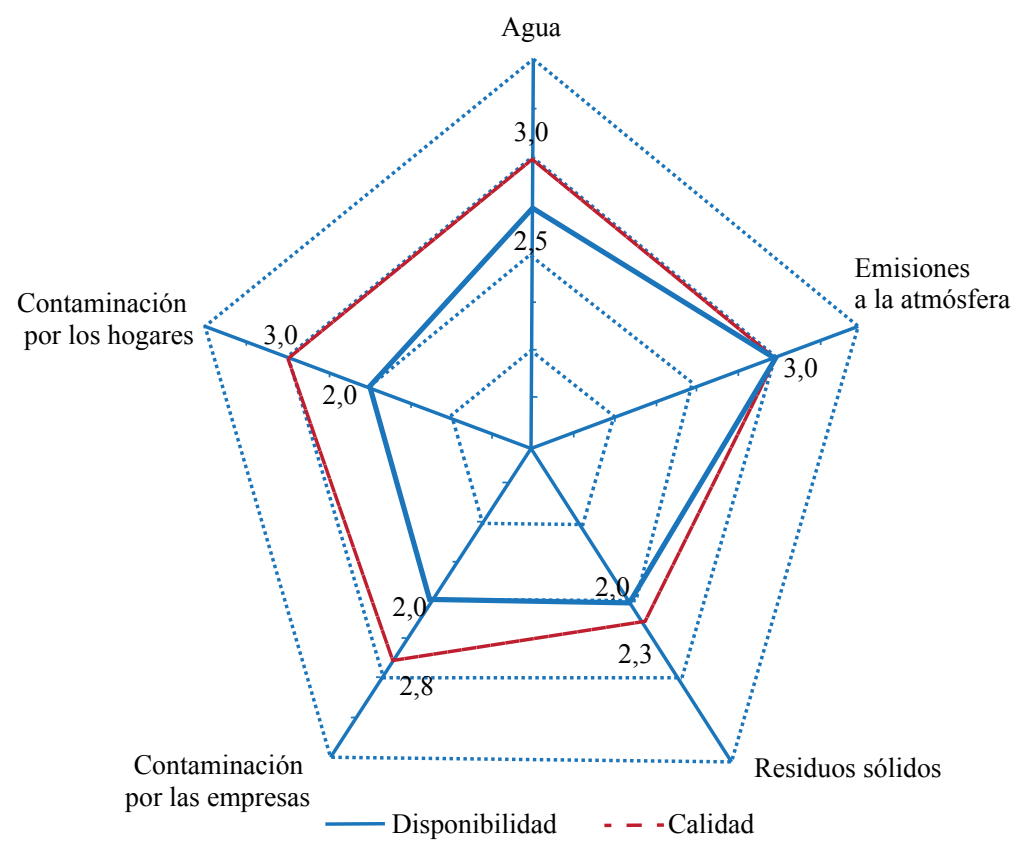

Fuente: Cuadro 4. 


\section{Bibliografía}

Adelson, J. y McCoach, B. (2010). Measuring the mathematical attitudes of elementary students: The effects of a 4-point or 5-point Likert-type scale. Educational and Psychological Measurement, 70(5), 796807. Recuperado de https://journals.sagepub.com/doi/abs/10.1177/00 13164410366694

Cámara de Diputados. (2014). Ley del Sistema Nacional de Información Estadística y Geográfica. Ciudad de México: Cámara de Diputados del H. Congreso de la Unión. Recuperado de http://www.diputados.gob.mx/ LeyesBiblio/ref/lsnieg.htm

Carifio, J. y Perla, R. (2007). Ten common misunderstandings, misconceptions, persistent myths and urban legends about Likert scales and Likert response formats and their antidotes. Journal of Social Sciences, 3(3), 106-116. Recuperado de https://thescipub.com/PDF/ jssp.2007.106.116.pdf

Clason, D. y Dormody, T. (1994). Analyzing data measured by individual Liker-type items. Journal of Agricultural Education, 35(4), 3135. Recuperado de http://citeseerx.ist.psu.edu/viewdoc/download? doi=10.1.1.113.2197\& $\mathrm{rep}=$ rep1\&type $=$ pdf

Conagua. (2017). Estadísticas del agua en México. Ciudad de México: Secretaría de Medio Ambiente y Recursos Naturales / Comisión Nacional del Agua. Recuperado de http://sina.conagua.gob.mx/publicaciones/ EAM 2017.pdf

Galindo, L. M. (2009). La economía del cambio climático en México: síntesis. Ciudad de México: Secretaría de Hacienda y Crédito Público / Secretaría de Medio Ambiente y Recursos Naturales. Recuperado de http://www.atl.org.mx/images/docs/Sintesis2009cambioclimatico.pdf

Guevara Sanginés, A. y Lara Pulido, J. A. (2014). Diagnóstico de la situación actual de las estadísticas ambientales en América Latina y el Caribe (Documento de Investigación). Ciudad de México: Banco Interamericano de Desarrollo (BID) / CEPAL / INEGI.

INECC. (2018). Inventario nacional de gases de efecto invernadero 2015. Ciudad de México: Instituto Nacional de Ecología y Cambio Climático. Recuperado de https://www.gob.mx/inecc/acciones-y-programas/ inventario-nacional-de-emisiones-de-gases-y-compuestos-de-efecto -invernadero

INEGI. (2008). VII Reunión Nacional de Estadística. Aguascalientes, Ags., México: Instituto Nacional de Estadística y Geografía. Recuperado de 
https://www.inegi.org.mx/eventos/otros/2008/rne/docs/Pdfs/Memoria _VII_RNE.pdf

INEḠI. (2018a). Estadistica. Datos. Medio ambiente. Secciones sobre agua, prácticas ambientales y residuos sólidos. Aguascalientes, Ags., México: Instituto Nacional de Estadística y Geografía. Recuperado de http://www.beta.inegi.org.mx/datos/ (consulta: 28 de junio de 2018).

INEGI. (2018b). Módulo de Hogares y Medio Ambiente (Mohoma), 2017. Aguascalientes, Ags., México: Instituto Nacional de Estadística y Geografía. Recuperado de https://www.inegi.org.mx/programas/mohoma /2017/ (consulta: 28 de junio de 2018).

INEGI. (2018c). Sistema de Consulta de Estadisticas Ambientales. Aguascalientes, Ags., México: Instituto Nacional de Estadística y Geografía. Recuperado de http://mapserver.inegi.org.mx/ambiental/map/ indexV3_FF.html (consulta: 28 de junio de 2018).

INEGI (2020). Inicio. Temas. PIB y cuentas nacionales. Satélite. Ecológicas. Aguascalientes, Ags., México, Instituto Nacional de Estadística, y Geografía. Disponible en https://www.inegi.org.mx/temas/ee/ (consulta: 28 de febrero de 2020).

Likert, R. (1932). A technique for the measurement of attitudes. Archives of Psychology, 140, 5-55. Recuperado de https://legacy.voteview.com/ pdf/Likert_1932.pdf

López Pérez, C. R. (2014). Estrategias de coordinación para impulsar las fuentes estadísticas de la información ambiental en México. En A. Mercado García y C. R. López Pérez (eds.), La estadística ambiental en México (pp. 555-576). Ciudad de México: El Colegio de México, A.C. / CEPAL.

Mediavilla Sahagún, A. (2014). México ante el cambio climático. En A. Mercado García y C. R. López Pérez (eds.), La estadística ambiental en México (pp. 437-456). Ciudad de México: El Colegio de México, A.C. / CEPAL.

Mercado García, A. y López Pérez, C. R. (2014), La estadística ambiental en México. Ciudad de México: El Colegio de México, A.C. / CEPAL.

Naciones Unidas. (1985). Un esquema para la elaboración de estadísticas del medio ambiente. Informes estadísticos (Documento de Investigación, serie M, núm. 78). Nueva York: Naciones Unidas.

Naciones Unidas. (2012). Framework for the Development of Environment Statistics. United Nations Economic and Social Council, Statistical Commission, 43rd session. Nueva York, del 28 de febrero al 2 de marzo. Recuperado de https://unstats.un.org/unsd/statcom/43rd-session/ documents/statcom-2012-43rd-report-E.pdf 
Naciones Unidas. (2013). Framework for the Development of Environment Statistics (FDES) 2013. United Nations Statistical Division, Statistical Commission, 44th session. Nueva York, 26 de febrero al 1 de marzo. Recuperado de http://unstats.un.org/unsd/statcom/doc13/ BG-FDES-Environment.pdf

OCDE. (2005). OECD Environmental indicators 2005 (Environment at a glance). París: Organización para el Desarrollo y la Cooperación Económicos. Recuperado de https://www.oecd-ilibrary.org/environment/ environment-at-a-glance 9789264012196-en

Quiroga, R. (2005). Estadísticas del medio ambiente en América Latina y el Caribe: avances y perspectivas (serie Manuales, núm. 43). Santiago de Chile: Comisión Económica para América Latina y el Caribe. Recuperado de https://repositorio.cepal.org/bitstream/handle/11362/5609/1/ S05629_es.pdf

Rapport, D. y Friend, A. (1979). Towards a comprehensive framework for environment statistics: A stress-response approach. Ottawa: Statistics Canada.

Schuschny, A. y Soto, H. (2009). Guía metodológica. Diseño de indicadores compuestos de desarrollo sostenible (Documento de trabajo). Comisión Económica para América Latina y el Caribe, Naciones Unidas. Recuperado de https://repositorio.cepal.org/bitstream/handle /11362/3661/S2009230_es.pdf?sequence=1\&isAllowed=y

Semarnat. (2009). Cambio climático. Ciencia, evidencia y acciones. Ciudad de México: Secretaría de Medio Ambiente y Recursos Naturales (serie ¿Y el medio ambiente?). Recuperado de https://es.slideshare.net/ Rakakakata/cambio-climtico-ciencia-evidencia-y-acciones-semarnat

Semarnat. (2014). Versión de difusión del Programa Especial de Cambio Climático, 2014-2018 (PECC 2014-2018). Gobierno de la República, Ciudad de México: Secretaría de Medio Ambiente y Recursos Naturales. Recuperado de https://www.gob.mx/cms/uploads/attachment/file/42488/ Programa_especial_de_cambio_climatico_2014-2018_vdifusion.pdf

Semarnat. (2018a). Registro de Emisiones y Transferencias de Contaminantes, RETC. Ciudad de México: Secretaría de Medio Ambiente y Recursos Naturales. Recuperado de http://apps1.semarnat.gob.mx/retc/retc/ index.php

Semarnat. (2018b). Sistema Nacional de Información Ambiental y de Recursos Naturales (SNIARN). Base de datos estadísticos. Badesniarn. Ciudad de México: Secretaría de Medio Ambiente y Recursos Naturales. Recuperado de http://dgeiawf.semarnat.gob.mx:8080/approot/ dgeia_mce/html/mce_index.html 
Sullivan, G. y Artino Jr., A. (2013). Analyzing and interpreting data from Likert-type scales. Journal of Graduate Medical Education, 5(4), 541-542. Recuperado de https://www.ncbi.nlm.nih.gov/pmc/articles/ PMC3886444/

Summers, G. F. (1970). Attitude measurement. Chicago, Il: Rand McNally [también publicado en español (1984), Medición de actitudes, Ciudad de México: Trillas].

Taboulchanas, K. y Fernández, F. (2009). Estado de la situación de las estadísticas ambientales en América Latina y el Caribe en 2008: avances, desafios y perspectivas (serie Estudios Estadísticos y Prospectivos, núm. 67). Santiago de Chile: CEPAL. Recuperado de https://repositorio. cepal.org/handle/11362/4771

\section{Acerca de los autores}

Alfonso Mercado García es coordinador del Programa de Análisis Económico de México (PRAEM) de El Colegio de México, A.C., institución donde además es profesor-investigador en el Centro de Estudios Económicos, y responsable del cuerpo académico "Políticas de industrialización y desarrollo: los casos de México y China". Cuenta con dos grados de maestría, uno en Economía, por El Colegio de México, y otro en Economía del Desarrollo, por la University of Sussex, Inglaterra. Es miembro del Sistema Nacional de Investigadores -desde hace 27 años, ininterrumpidamente- y en la actualidad es nivel II. Es autor y compilador de diez libros, así como autor de varios capítulos de libros y artículos sobre economía aplicada. ORCID: https://orcid.org/0000-0003-3789-5920

Entre sus publicaciones se encuentran:

Mercado, A. (2010). Los compromisos adquiridos en acuerdos de libre comercio bilaterales y multilaterales y los espacios para una politica industrial en México y Centroamérica. Ciudad de México: Comisión Económica para América Latina y el Caribe (CEPAL), Sede Subregional en México (2010-92, LC/MEX/L.999). Disponible en https:// repositorio.cepal.org/handle/11362/26041

Mercado, A., Miyamoto, K. y O'Connor, D. (eds.). (2008). Inversión extranjera directa, tecnología y recursos humanos en los países en desarrollo. Ciudad de México: El Colegio de México, A.C. / París, Francia: Centro de Desarrollo, OCDE. 
Carlos Roberto López Pérez es maestro en Desarrollo Urbano por El Colegio de México, A.C. (Colmex) y es licenciado en Sociología por la Universidad Nacional de El Salvador. Fue investigador en el Programa de Estudios Energéticos del Colmex (1981-1988), donde realizó (en coautoría) dos libros, además de varios ensayos sobre el tema. Fungió como director de Estadísticas del Medio Ambiente en el INEGI, donde realizó investigación, y participó como autor o coordinador en varias publicaciones y artículos al respecto (en el ámbito nacional y por zonas metropolitanas), así como sobre sustentabilidad. También ha participado en grupos de trabajo sobre medio ambiente e indicadores de desarrollo sustentable, patrocinados por la Organización de las Naciones Unidas. ORCID: https://orcid.org/00000001-6256-0212

Entre sus publicaciones se encuentran:

Sordo, A. M. y López, C. R. (1988). Exploración, reservas y producción de petróleo en México, 1970-1985. Ciudad de México: El Colegio de México, A.C., Programa de Energéticos.

Alonso Palacios, A. y López, C. R. (1986). El Sindicato de Trabajadores Petroleros y sus relaciones con Pemex y el Estado, 1970-1985. Ciudad de México: El Colegio de México, A.C., Programa de Energéticos.

Recepción: 17 de agosto de 2018. Aceptación: 23 de enero de 2019. 
